\title{
Regulation of GTP cyclohydrolase I by alternative splicing in mononuclear cells
}

\author{
Wuh-Liang Hwu, ${ }^{\text {a }}$ Hui-Ying Yeh, ${ }^{\text {a }}$ Shu-Wen Fang, ${ }^{\text {a }}$ Hao-Sen Chiang, \\ Yu-Wei Chiou, ${ }^{b}$ and Yu-May Lee ${ }^{b, *}$ \\ a Department of Pediatrics and Medical Genetics, National Taiwan University Hospital and National Taiwan University, \\ College of Medicine, Taipei, Taiwan, ROC \\ ${ }^{\mathrm{b}}$ Institute of Biological Chemistry, Academia Sinica, and Institute of Biochemical Science, National Taiwan University, Taipei, Taiwan, ROC
}

Received 22 May 2003

\begin{abstract}
GTP cyclohydrolase I ( $\mathrm{GCH}$, EC 3.5.4.16) regulates the level of tetrahydrobiopterin and in turn the activities of nitric oxide synthase and aromatic amino acid hydroxylases. Type II GCH mRNA, an alternatively spliced species abundant in blood cells, encodes a truncated and nonfunctional protein. When we stimulate peripheral blood mononuclear cells by PHA, the transcription of full-length GCH mRNA increased, but that of type II mRNA decreased transiently. We further demonstrated that the type II cDNA exerted a dominant-negative effect on the wild-type cDNA, similar to the effect of some GCH mutants. Therefore, type II mRNA may regulate $\mathrm{GCH}$ and then contribute to the regulation of NO production by BH4-dependent iNOS in mononuclear cells. Selection of the splicing sites may be coupled with transcriptional activation of the GCH gene.
\end{abstract}

(C) 2003 Elsevier Science (USA). All rights reserved.

Keywords: GTP cyclohydrolase I; Alternative splicing; Dominant-negative effect; Regulation

Tetrahydrobiopterin $\left(\mathrm{BH}_{4}\right)$ is the essential cofactor of phenylalanine hydroxylase, tyrosine hydroxylase, tryptophan hydroxylase, and nitric oxide synthase (NOS). The rate-limiting step of $\mathrm{BH}_{4}$ biosynthesis is the cleavage of GTP to 7,8-dihydroneopterin triphosphate by the enzyme GTP cyclohydrolase I (GCH, EC 3.5.4.16) [1]. $\mathrm{GCH}$ is therefore necessary for the synthesis of the signaling molecules dopamine, norepinephrine, epinephrine, serotonin and nitric oxide as well as the detoxification of the amino acid L-phenylalanine [2-4].

Mutations of the GCH gene induce human diseases including malignant hyperphenylalaninemia (HPA) and dopa-responsive dystonia (DRD) [5]. HPA is induced by recessive $\mathrm{GCH}$ mutations and is associated with hyperphenylalaninemia, dystonia, seizure, and neurological

\footnotetext{
Abbreviations: GCH, GTP cyclohydrolase I; $\mathrm{BH}_{4}$, tetrahydrobiopterin; NOS, nitric oxide synthase; HPA, hyperphenylalaninemia; DRD, dopa-responsive dystonia; DAHP, 2,4-diamino-6-hydroxypyrimidine.

${ }^{*}$ Corresponding author. Fax: +886-2-2788-9759.

E-mail address: YML6120@gate.sinica.edu.tw (Y.-M. Lee).
}

degeneration, probably due to the lack of both catecholamines (dopamine, noradrenaline, and adrenaline) and serotonin [6]. Dystonia is the only symptom in DRD patients and the response to low-dose L-dopa is excellent. DRD is often caused by dominant GCH mutations with residual $\mathrm{GCH}$ activities up to $15 \%$ of normal [7-9]. $\mathrm{GCH}$ protein is a multimeric protein composed of 10 identical subunits [10], and we have demonstrated that some dominant GCH mutations exerted a dominant-negative effect toward the wild-type GCH protein [11].

Besides the control of neurotransmitter synthesis, $\mathrm{BH}_{4}$ is a determining factor for NOS activity. GCH expression is enriched in brain, liver, and endothelial cells, and is inducible in other cell types and tissues. $\mathrm{BH}_{4}$ synthesis and NOS are co-induced by proinflammatory cytokines [12]. Experiments employing GCH inhibitor 2,4-diamino-6-hydroxypyrimidine (DAHP) demonstrated that $\mathrm{BH}_{4}$ is a limiting factor of nitric oxide generation in experimental endotoxic shock in rat [13]. Stabilization of $\mathrm{BH}_{4}$ by L-ascorbic acid potentiates endothelial nitric oxide synthesis [14]. It has been shown 
that cis-elements required for cell type-specific cAMPdependent enhancement of gene transcription are located along the GCH core promoter [15].

There are two alternatively spliced GCH mRNAs (types II and III) reported initially [16]. These mRNAs are identical at their $5^{\prime}$-regions, but diverge just after the break point between exons 5 and 6 . The full-length (type I) mRNA contains a long $3^{\prime}$-noncoding region encoded by exon 6 [17]. A splicing between the $5^{\prime}$ donor site of intron 5 and the middle of exon 6 generates type II mRNA. Intron 5 is not spliced in type III mRNA. Two more types, types IV and V, of alternative splicing were reported in human myelomonocytoma cells (THP-1) [18]. In this paper, we studied alternative splicing of GCH mRNAs and their role in human peripheral blood mononuclear cells. We proved the previously described forms and demonstrated a new form of alternative splicing. We characterized the transcription of these mRNAs when blood cells were stimulated and conferred a role upon the type II mRNA in CGH regulation.

\section{Materials and methods}

Cells and cultures. BHK, HepG2, HeLa, Huh7, and T24 (bladder transitional-cell carcinoma) cells were obtained from the American Type Culture Collection directly or through the National Health Research Institute Cell Bank. HepG2, HeLa, and Huh7 cells were grown in Dulbecco's minimal essential medium (DMEM) with 10\% (v/v) fetal-calf serum (FCS) (Gibco, USA). T24 cells were grown in Eagle's minimal essential medium (MEM) with Earle's salts supplemented with 10\% (v/v) heat-inactivated FCS (Gibco, USA). Confluent monolayer of T24 cells stimulated with $0-1000 \mathrm{IU} / \mathrm{ml}$ IFN- $\gamma$ (PeproTech, England) in fresh medium for $24 \mathrm{~h}$. Peripheral blood mononuclear cells were obtained by centrifuging diluted blood (1 part of blood +1 part of phosphate-buffered saline) over Ficoll-Paque (Amersham-Pharmacia Biotech, USA). The interface region was separated and washed. Mononuclear cells purified from $20 \mathrm{ml}$ blood were suspended in $30 \mathrm{ml}$ RPMI 1640 (Gibco, USA) with $15 \%$ heat-inactivated FCS and then split into six T25 flasks. Peripheral blood mononuclear cells were stimulated by PHA (Murex, France) for up to $72 \mathrm{~h}$. Nitrite concentrations in the culture medium were determined by the Griess reagent (Promega, USA).

Construction of plasmids. GCH cDNA was kindly provided by Dr. Nagatsu. This cDNA produced functional protein although it lacks a short $5^{\prime}$ piece [19]. Type II cDNA was produced by PCR using GCH cDNA as a template and cloned into a CMV promoter expression vector (pCMV-GCH-II). pCMV-A16-GCH-wt was constructed by inserting full-length GCH cDNA (NarI/EcoRI fragment) into pCMVAGP/EBP (replacing its Bst $\mathrm{BI} / E c o$ RI fragment) [20]. This generated an $\mathrm{N}$-terminal AGP/EBP A16 epitope. The construction of pCMV-A16GCH-249 and pCMV-A16-GCH-201 has been described [9,11].

Detection of $G C H$ alternative splicing by reverse transcription-coupled PCR. Cytoplasmic RNA was extracted from cells with a Total RNA Extraction Miniprep System (Viogene, Taiwan). For the measurement of GCH mRNA transcribed from transfected plasmids, mRNA was extracted by a QuickPrep Micro mRNA Purification kit (Amersham-Pharmacia Biotech, USA). The upper strand PCR primer used was F2 (5'-GCCCCGCAGCGAGGAGGATAAC) and lower strand primers were R3 (5'-GACAGACAATGCTACTGGCAGT) for the full-length transcript and S1 (5'-GAAGCTATGGTTCTGCA GAC) for the spliced forms (Fig. 1).

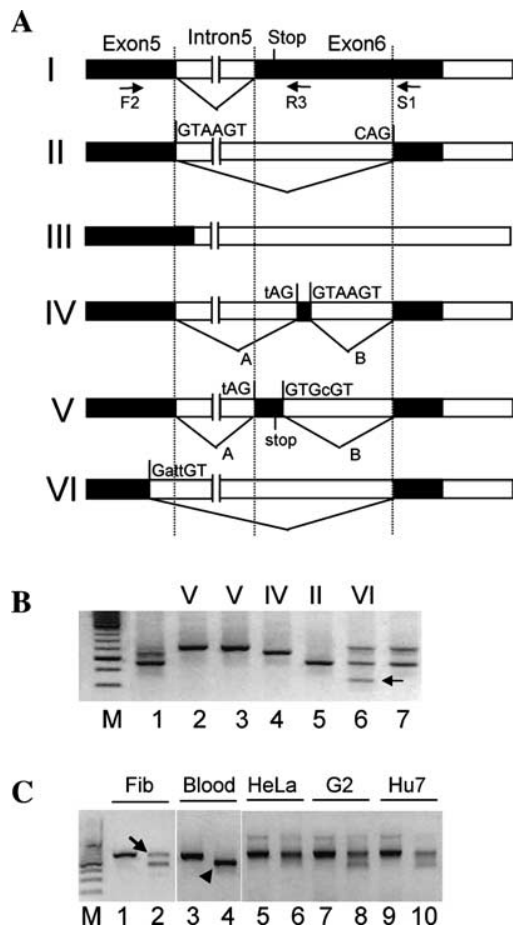

Fig. 1. (A) A schematic sketch of six types of GTP cyclohydrolase I gene alternative splicing. The dark boxes indicate sequences that are included in the mRNA. The position of the authentic stop codon is marked (Stop). The arrows indicate the positions of primers used in RT-PCR. (B) Amplification of GCH mRNAs from diluted template, which shows the presence of type V (lanes 2 and 3), type IV (lane 4), type II (lane 5), and type VI mRNA (lane 6, arrow). M denotes molecular weight markers. (C) Abundance of GCH mRNAs in cells. Lanes 1, 3, 5, 7, and 9 are amplifications for the full-length mRNA and lanes 2, 4, 6, 8, and 10 are spliced forms. Lanes 1 and 2 are from human skin fibroblast (Fib); lanes 3 and 4, human peripheral blood mononuclear cells (Blood); lanes 5 and 6 , HeLa cells (HeLa); lanes 7 and 8, HepG2 cells (G2); and lanes 9 and 10, Huh7 cells (Huh7). The major spliced mRNAs are type IV (arrow) and type II (arrow head).

LightCycler PCR. Quantitative PCRs were performed in a LightCycler system (Roche Molecular Biochemicals, Mannheim, Germany) [21]. In glass capillaries with a total volume of $20 \mu$, the reaction mixture consisted of $2 \mu \mathrm{l}$ of FastStart DNA Master SYBR Green I (Roche Diagnostics GmbH, Mannheim, Germany), $0.5 \mu \mathrm{M}$ each of the primers, $3 \mathrm{mM} \mathrm{MgCl}_{2}$, and $2 \mu \mathrm{l} \mathrm{cDNA}$ after a 1:10 dilution. In each run, one negative control, with water added instead of DNA, was included. Primers used for type I mRNA were GCH-SPL-1 $\left(5^{\prime}\right.$ GGTTCCATTTGTTGGAAAGG) and GCH-SPL-2 (5'-CACA TGTGTGTTGCTTCAACC); for type II were GCH-SPL-1 and GCH-SPL-3 (5'-GGTTCTGCAGACGTTGCTTC); and for type III were GCH-SPL-1 and GCH-SPL-4 (5'-TGCAGACTTACGTTGC TTCAA). All lower strand primers were designed to cross the splicing junctions. Human GAPD gene was used to normalize the cDNA levels. A standard thermal cycler program suggested by the manufacturer was used except that the annealing temperature was $60^{\circ} \mathrm{C}$. Concentrations were calculated by Roche LightCycler software ver3.5, with standard curves made by serial dilution of PCR fragments from $2.33 \times 10^{-1}$ to $2.33 \times 10^{-9} \mathrm{ng} / \mu \mathrm{l}$ (type I) and from $2.65 \times 10^{-1}$ to $2.65 \times 10^{-9} \mathrm{ng} / \mu \mathrm{l}$ (type II) per reaction.

Transfection and Western blot analysis. Calcium phosphate precipitation method was used for transfection [22]. BHK cells in $6 \mathrm{~cm}$ dishes were transfected with a combination of pCMV-A16-GCH-wt, pCMV-GCH-II, and pCMV. Forty hours after transfection, cells were 
subjected to either mRNA extraction or Western blot analysis. Western blot analysis was performed with a $\mathrm{GCH}$ antiserum [9] by standard methods. Blots were developed using the ECL detection system (Amersham-Pharmacia Biotech, USA).

Amplification of splicing factors. Primers for RT-PCR of splicing factor SC-35 [23] were 5'-GAACATCTACAACGCCTGC and 5'ACGTTCGGAACGGAAAGG; SF2 [24] 5'-GTTTACCGAGAT GGCACTG and 5'-ATCAAAGACACGAAGGGAATG; hnRNP-A1 [24] 5'-GAATGCAAGGCCACACAAG and 5'-ACCATATCCAC CACCACCAC; PTB [25] 5'-GAACATCTACAACGCCTGC and 5'ACGTTCGGAACGGAAAGG; SRP30C [26] 5'-TTCAGGACT TCCTCCGTCAG and 5'-CACAAAGCAGCTCAGTTAACC; and Htra-2 $\beta 1$ [27] 5'-AGACCACATACGCCAACAC and 5'-GCCCA CAAACAATATCCCAG.

\section{Results}

\section{GCH gene alternative splicing}

The six types of GCH alternative splicing are depicted in Fig. 1A. Types I to III mRNAs have been reported by Togari et al. [16] in liver, types IV and V mRNAs have been reported by Golderer et al. [18] in human myelomonocytoma cells, and type VI has not been described (GenBank AY137465). Type IV mRNA encodes a truncated protein. Type $\mathrm{V}$ mRNA contains the authentic stop codon thus encodes a full-length $\mathrm{GCH}$ protein. Type VI is less abundant, which uses an alternative $5^{\prime}$ donor site within exon 6 , and the same $3^{\prime}$ acceptor site as in type II splicing. These mRNAs were also obtained separately by the amplification of diluted template (Fig. 1B) and were proved by direct sequencing.

\section{Abundance of GTP cyclohydrolase I $m R N A$ s in different types of cells}

Since GCH is enriched in brain, liver, and endothelial cells, and is inducible in other cell types, we verify the spliced forms in different cells. Cytoplasmic RNA was extracted from human skin fibroblasts and cell lines, and the full-length and alternatively spliced GCH mRNAs were amplified separately (Fig. 1C). Besides the fulllength $\mathrm{GCH}$ gene transcript, human skin fibroblasts expressed two major forms of the alternatively spliced mRNAs, type IV (arrow) and II; human peripheral blood mononuclear cells expressed predominantly the shorter type II mRNA (arrowhead); HeLa cells expressed predominantly the type IV mRNA; and HepG2 and Huh7 cells the type IV and a variable amount of type II mRNA (Fig. 2). Type V mRNA is less abundant and type VI mRNA is the rarest.

\section{Differential induction of GCH transcripts in blood mono- nuclear cells by PHA}

GCH activity has been routinely assayed in peripheral blood mononuclear cells after PHA stimulation, because the basal GCH activity in blood cells was low
A

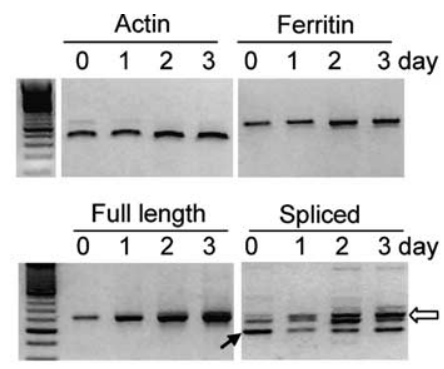

B
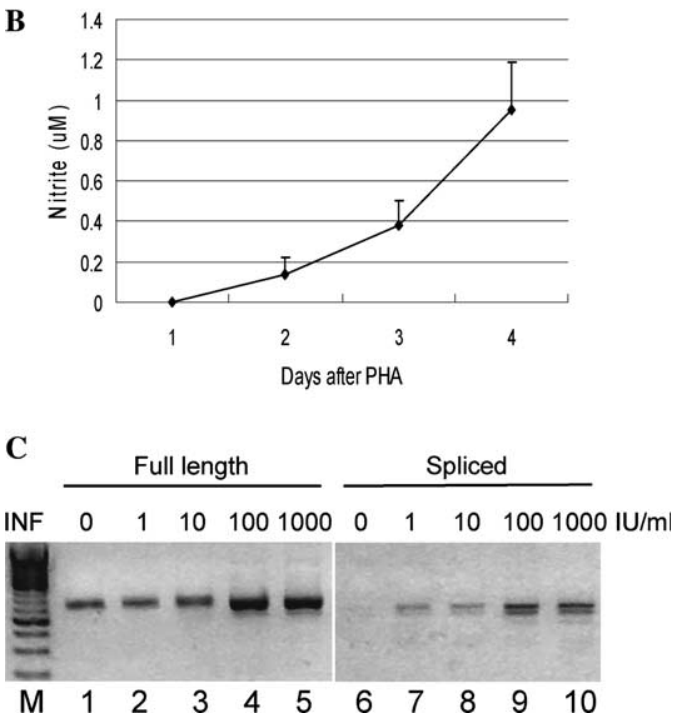

Fig. 2. Induction of GCH mRNAs. (A) Peripheral blood mononuclear cells were treated with PHA and harvested on days $0,1,2$, and 3. RTPCR was done for actin, ferritin, full-length GCH mRNA, and spliced GCH mRNAs. (B) Increase of nitrite concentration in culture mediums during the stimulation of blood cells by PHA. The bars on the data points indicate one standard deviation. (C) T24 cells treated with $0-1000 \mathrm{IU}$ IFN- $\gamma / \mathrm{ml}$ for $24 \mathrm{~h}$ and GCH mRNAs amplified.

[28]. Under the stimulation of PHA, cell proliferation occurred as shown by the measurement of actin and ferritin mRNAs, however the full-length GCH mRNA increased to a much greater extent (Fig. 2A). Before PHA stimulation, type II mRNA (Fig. 2A, arrow) was the major spliced form in mononuclear cells, but its amount decreased paradoxically after PHA stimulation. On the contrary, the type IV and type V mRNA (Fig. 2A, open arrow) increased along with the fulllength transcript. Nitrite accumulated in the culture medium after the cells were stimulated by PHA (Fig. 2B). In T24 cells, the type IV mRNA can also be stimulated by INF- $\gamma$ along with the induction of the full-length transcript (Fig. 2C). Nevertheless, there was no visible type II mRNA both before and after INF- $\gamma$ treatment. Therefore, the expression and induction of $\mathrm{GCH}$ mRNAs is cell type specific.

\section{Quantification of the spliced forms of GCH transcripts}

The quantities of types I, II, and III GCH mRNAs were determined by the LightCycle PCR. Before 
treatment, the concentration of type II mRNA in mononuclear cells was higher than that of type I mRNA. One day after PHA stimulation, the concentration of type I mRNA increased, but that of type II mRNA decreased (Fig. 3A). While the concentrations of GCH mRNAs differed significantly between each independent experiment, when we calculated the changes (fold increase) of mRNA quantities, the tendency was pretty constant (Fig. 3B). The concentration of type III mRNA was much lower than that of either type I or type II mRNA (data not shown).

\section{Expression of the type II cDNA decreases the level of the wild-type GCH protein}

Some GCH mutants exert dominant-negative effects on the wild-type protein [11], and the type II mRNA encodes a truncated protein with no enzyme activity [29]. When wild-type and type II cDNAs were introduced simultaneously into BHK cells by calcium phosphate precipitation method, the presence of type II cDNA decreased the level of wild-type $\mathrm{GCH}$ protein (Fig. 4A, lanes 1-4, and lanes 9 and 10). In the control experiments, GCH mutant G201E (lanes 11 and 12), but not R249S (lanes 7 and 8), also disclosed a dominantnegative effect. Type II GCH cDNA itself did not result
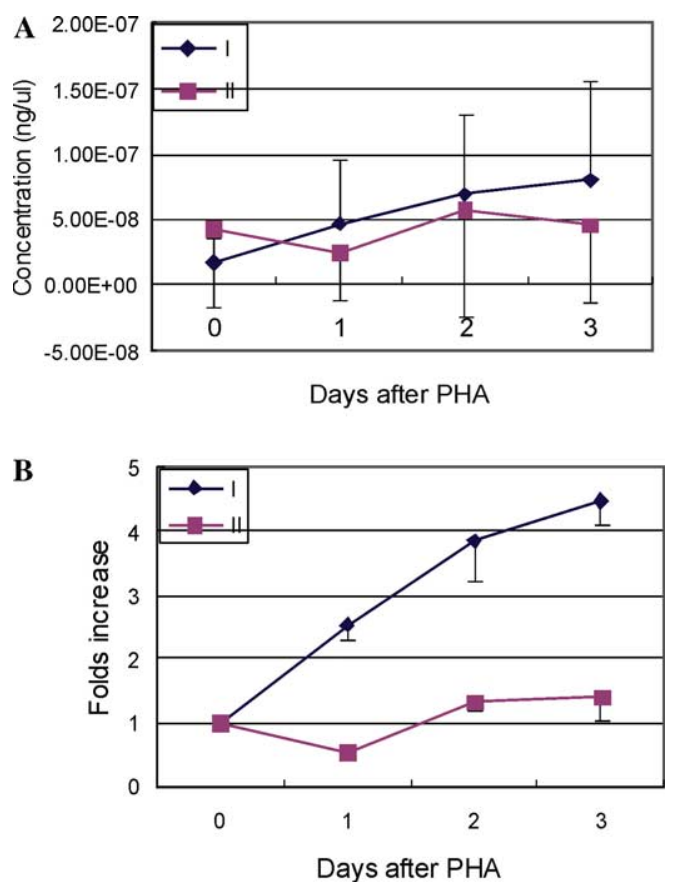

Fig. 3. Quantitative RT-PCR for GCH types I and II mRNA. Peripheral blood mononuclear cells were treated with PHA and harvested on days $0,1,2$, and 3. (A) The concentrations of mRNAs. (B) Fold increases of GCH mRNAs after the treatment of cells by PHA. The bars on the data points indicate one standard deviation calculated from three independent experiments. in any visible $\mathrm{GCH}$ protein (lane 13). The levels of mRNA expressed from the transfected vectors were similar (Fig. 4B).

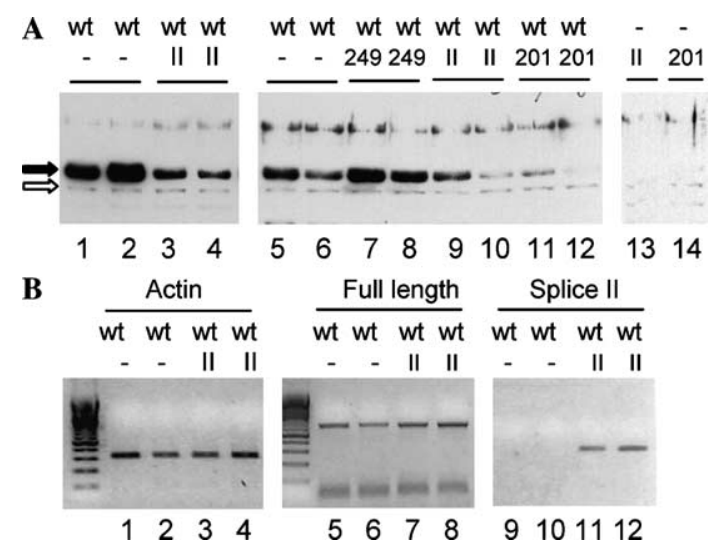

Fig. 4. Expression of GCH cDNAs in BHK cells. (A) Western blot analysis with $\mathrm{GCH}$ antibody. Wild-type GCH plasmid pCMV-A16GCH-wt (wt) $(0.8 \mu \mathrm{g}$ in lanes $1-4$ and $0.6 \mu \mathrm{g}$ in other lanes) was transfected into BHK cells together with the spliced-form plasmid pCMV-GCH-II (II) $(1.8 \mu \mathrm{g}$ in lanes 3 and 4 , and $0.6 \mu \mathrm{g}$ in other lanes), R249S mutant plasmid $(249,0.6 \mu \mathrm{g})$, or G201E mutant plasmid (201, $0.6 \mu \mathrm{g})$. The arrow indicates the A16-tagged $\mathrm{GCH}$ protein and the open arrow indicates a cross-reacting band. (B) RT-PCR for actin (lanes 1-4), full-length GCH mRNA (lanes 5-8), and type II GCH mRNA (lanes 9-12) in BHK cells to show transcription of the transfected vectors.
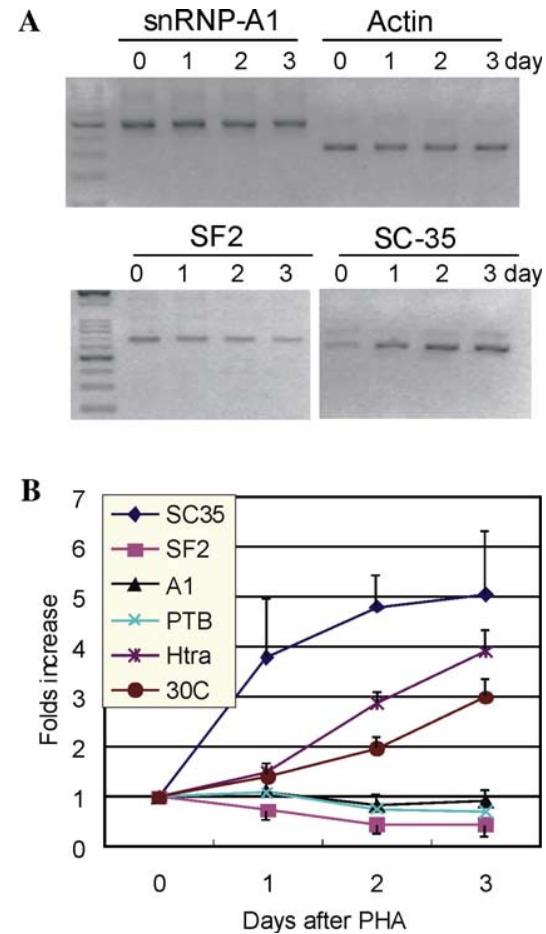

Fig. 5. Transcription of splicing factors by PHA treatment. Peripheral blood mononuclear cells were treated with PHA and harvested on days $0,1,2$, and 3. (A) RT-PCR was performed for hnRNP-A1, actin, SF2, and SC-35. (B) Data normalized by actin mRNA for hnRNP-A1 (A1), SF2, SC-35, PTB, Htra-2 31 (Htra), and SRP30C (30C). The bars on the data points indicate one standard deviation. 
Changes in splicing factor expression after PHA stimulation

From the results presented in the previous sections, it is clear that the selection of splicing points changes when peripheral blood mononuclear cells were stimulated by PHA. Therefore, we measured the transcription of several splicing factors by semiquantitative RT-PCR. The data revealed that SC-35, Htra-2 $\beta 1$, and SRP30C were induced, hnRNP-A1 and PTB stayed steadily, while the transcription of SF2 was repressed by PHA (Fig. 5A). The data were also quantified and normalized by the levels of actin mRNA (Fig. 5B).

\section{Discussion}

Alternative splicing is a major mechanism to enable a single gene to increase its coding capacity [30], to facilitate its tissue-specific function [31], or to contribute to its regulation (as revealed in this study). In this study, we demonstrated complex alternative splicing forms over the $3^{\prime}$-end of the GCH gene. Along with the increase in the full-length GCH transcript, the type II mRNA decreased by PHA treatment, showing a fine-tune regulation for GCH in mononuclear cells.

GCH catalyzes the rate-limiting step of $\mathrm{BH}_{4}$ biosynthesis, and the induction of GCH leads to an elevation in tissue $\mathrm{BH}_{4}$ concentration, which in turn contributes to the syntheses of neurotransmitters and nitric oxide to cope with acute stress to the body. Among the alternatively spliced GCH mRNA species, the type II mRNA is abundant in several tissues and cells ([18] and this study). It encodes a nonfunctional polypeptide lacking the C-terminal part of $\beta$-sheet domain. Since the $\beta$-sheet domain is responsible for both enzyme activity and complex formation, the type II mRNA does not give rise to active enzyme [29]. Our results demonstrated that type II cDNA could exert a dominant-negative effect on the full-length $\mathrm{GCH}$ protein, so type II splicing may erratically decrease GCH activity. One would ask why this mRNA exists. In this study, the response of type II mRNA to PHA stimulation may help to resolve the question. In mononuclear cells, the production of NO by inducible NO synthase (iNOS) is important for the function of these cells during infection or inflammation. We hypothesize that the presence of type II mRNA contributes to the regulation of $\mathrm{GCH}$ by reducing its basal activity and then allowing a bigger amplitude of induction. Differential splicing of the GCH RNA has also been implicated in the variation of clinical symptoms in DRD [32].

When we compared the sequence at the splicing junctions, we found that the alternative $3^{\prime}$ acceptor site used by type II splicing actually fits the consensus better than that used by the full-length splicing (Fig. 1A). This could explain the abundance of the type II mRNA. It is intriguing that how these two $3^{\prime}$ acceptor sites are selected in different conditions. The heterogeneous nuclear ribonucleoproteins (hnRNPs) have been implicated in splicing repression [33]. The SR proteins, which are required for constitutive splicing, also influence alternative splicing regulation [34]. The SR protein SF2/ASF and hnRNP A1 could be functional antagonists in several examples [24]. In our study, we could observe a specific pattern of changes in mRNA for a panel of splicing factors, including the large induction of $\mathrm{SC}-35$, for which the "SC-35 domain" is a place where processing of many pre-mRNAs occurs. Besides, alternative splicing has also been shown to be coupled with the transcription machinery [35]. Since the existence and the role of alternative splicing are rapidly emerging, this study should contribute significantly to both the mechanism of $\mathrm{GCH}$ regulation and to the understanding of alternative splicing.

\section{Acknowledgments}

We thank Dr. Nagatsu for the GCH plasmid. This work was supported by National Science Council Grants NSC 90-2314-B-002163 and NSC 91-2314-B-002-147.

\section{References}

[1] C.A. Nichol, G.K. Smith, D.S. Duch, Biosythesis and metabolism of tetrahydrobiopterin and molybdopterin, Ann. Rev. Biochem. 54 (1985) 729-764.

[2] S. Kaufman, Studies on the mechanism of the enzymatic conversion of phenylalanine to tyrosine, J. Biol. Chem. 234 (1959) 2677-2682.

[3] T. Nagatsu, M. Levitt, S. Udenfriend, Tyrosine hydroxylase: the initial step in norepinephrine biosynthesis, J. Biol. Chem. 239 (1964) 2910-2917.

[4] O.W. Griffith, J.S. Dennis, Nitric oxide synthase: properties and catalytic mechanism, Ann. Rev. Physiol. 57 (1995) 707-736.

[5] B. Thony, N. Blau, Mutations in the GTP cyclohydrolase I and 6pyruvoyl-tetrahydropterin synthase genes, Hum. Mutat. 10 (1997) 11-20.

[6] A. Niederwieser, N. Blau, M. Wang, P. Joller, M. Atarés, J. Cardesa-Garcia, GTP cyclohydrolase I deficiency, a new enzyme defect causing hyperphenylalaninemia with neopterin, biopterin, dopamine, and serotonin deficiencies and muscular hypotonia, Eur. J. Pediatr. 141 (1984) 208-214.

[7] M. Segawa, A. Hosaka, F. Migagawa, Y. Nomura, H. Imai, Hereditary progressive dystonia with marked diurnal fluctuation, in: R. Eldridge, S. Fahn (Eds.), Dystonia, Advance in Neurology, Raven, New York, 1976, pp. 215-233.

[8] H. Ichinose, T. Nagatsu, Molecular genetics of hereditary dystonia - mutations in the GTP cyclohydrolase I gene, Brain Res. Bull. 43 (1997) 35-38.

[9] W.L. Hwu, P.J. Wang, K.J. Hsiao, T.R. Wang, Y.M. Lee, Doparesponsive dystonia induced by recessive GTP cyclohydrolase I mutation, Hum. Genet. 105 (1999) 226-230.

[10] H. Nar, R. Huber, W. Meining, C. Schmid, S. Weinkauf, A. Bacher, Atomic structure of GTP cyclohydrolase I, Structure 3 (1995) 459-466. 
[11] W.L. Hwu, Y.W. Chiou, S.Y. Lai, Y.M. Lee, Dopa-responsive dystonia is induced by a dominant-negative mechanism, Ann. Neurol. 48 (2000) 609-613.

[12] A. Togari, M. Arai, M. Mogi, A. Kondo, T. Nagatsu, Coexpression of GTP cyclohydrolase I and inducible nitric oxide synthase mRNAs in mouse osteoblastic cells activated by proinflammatory cytokines, FEBS Lett. 428 (1998) 212-216.

[13] A.J. Bune, M.P. Brand, S.J. Heales, J.K. Shergill, R. Cammack, H.T. Cook, Inhibition of tetrahydrobiopterin synthesis reduces in vivo nitric oxide production in experimental endotoxic shock, Biochem. Biophys. Res. Commun. 220 (1996) 13-19.

[14] R. Heller, A. Unbehaun, B. Schellenberg, B. Mayer, G. WernerFelmayer, E.R. Werner, L-Ascorbic acid potentiates endothelial nitric oxide synthesis via a chemical stabilization of tetrahydrobiopterin, J. Biol. Chem. 276 (2001) 40-47.

[15] G. Kapatos, S.L. Stegenga, K. Hirayama, Identification and characterization of basal and cyclic AMP response elements in the promoter of the rat GTP cyclohydrolase I gene, J. Biol. Chem. 275 (2000) 5947-5957.

[16] A. Togari, H. Ichinose, S. Matsumoto, K. Fujita, T. Nagatsu, Multiple mRNA forms of human GTP cyclohydrolase I, Biochem. Biophys. Res. Commun. 187 (1992) 359-365.

[17] T. Nomura, M. Ohtsuki, S. Matsui, C. Sumi-Ichinose, H. Nomura, Y. Hagino, K. Iwase, H. Ichinose, K. Fujita, T. Nagatsu, Isolation of a full-length cDNA clone for human GTP cyclohydrolase I type 1 from pheochromocytoma, J. Neural. Transm. 101 (1995) 237-242.

[18] G. Golderer, E.R. Werner, C. Heufler, W. Strohmaier, P. Grobner, G. Werner-Felmayer, GTP cyclohydrolase I mRNA: novel splice variants in the slime mould Physarum polycephalum and in human monocytes (THP-1) indicate conservation of mRNA processing, Biochem. J. 355 (2001) 499-507.

[19] H. Ichinose, T. Ohye, Y. Matsuda, T. Hori, N. Blau, A. Burlina, B. Rouse, R. Matalon, K. Fujita, T. Nagatsu, Charaterization of mouse and human GTP cyclohydrolase I genes. Mutations in patients with GTP cyclohydrolase I deficiency, J. Biol. Chem. 270 (1995) 10062-10071.

[20] Y.M. Lee, L.W. Miau, C.J. Chang, S.C. Lee, Transcriptional induction of alpha-1 acid glycoprotein gene by synergistic interaction of two alternative activator forms of AGP/EBP (C/ EBP $\beta$ ) and NF-kB or Nopp140, Mol. Cell. Biol. 16 (1996) 4257-4263.

[21] C.T. Wittwer, K.M. Ririe, R.V. Andrew, D.A. David, R.A. Gundry, U.J. Balis, The LightCyclerk: a microvolume multisam- ple fluorimeter with rapid temperature control, Bio-Techniques 22 (1997) 176-181.

[22] F. Graham, A. van der Erb, A new technique for the assay of infectivity of human adenovirus 5 DNA, Virology 52 (1973) 456-457.

[23] X.D. Fu, T. Maniatis, Factor required for mammalian spliceosome assembly is localized to discrete regions in he nucleus, Nature 343 (1990) 437-444.

[24] A. Mayeda, A.R. Krainer, Regulation of alternative pre-mRNA splicing by hnRNP A1 and splicing factor SF2, Cell 68 (1992) 365-375.

[25] A. Gil, P.A. Sharp, S.F. Jamison, M.A. Garcia-Blanco, Characterization of cDNAs encoding the polypyrimidine tract-binding protein, Genes Dev. 5 (1991) 1224-1236.

[26] G.R. Screaton, J.F. Caceres, A. Mayeda, M.V. Bell, M. Plebanski, D.G. Jackson, J.I. Bell, A.R. Krainer, Identification and characterization of three members of the human SR family of premRNA splicing factors, EMBO 14 (1995) 4336-4349.

[27] R. Tacke, M. Tohyama, S. Ogawa, J.L. Manley, Human Tra2 proteins are sequence-specific activators of pre-mRNA splicing, Cell 93 (1998) 139-148.

[28] N. Blau, P. Joller, M. Atares, J. Cardesa-Garcia, A. Niederwieser, Increase of GTP cyclohydrolase I activity in mononuclear blood cells by stimulation: detection of heterozygotes of GTP cyclohydrolase I deficiency, Clin. Chim. Acta 148 (1985) 47-52.

[29] M. Gutlich, E. Jaeger, K.P. Rucknagel, T. Werner, W. Rodl, I. Ziegler, A. Bacher, Human GTP cyclohydrolase I: only one out of three cDNA isoforms gives rise to the active enzyme, Biochem. J. 15 (1994) 215-221.

[30] J.F. Caceres, A.R. Kornblihtt, Alternative splicing: multiple control mechanisms and involvement in human disease, Trends Genet. 18 (2002) 186-193.

[31] A. Hanamura, J.F. Caceres, A. Mayeda, B.R. Franza Jr., A.R. Krainer, Regulated tissue-specific expression of antagonistic premRNA splicing factors, RNA 4 (1998) 430-444.

[32] M. Hirano, Y. Imaiso, S. Ueno, Differential splicing of the GTP cyclohydrolase I RNA in dopa-responsive dystonia, Biochem. Biophys. Res. Commun. 234 (1997) 316-319.

[33] G. Dreyfuss, M.J. Matunis, S. Pinol-Roma, C.G. Burd, hnRNP proteins and the biogenesis of mRNA, Ann. Rev. Biochem. 62 (1993) 289-321.

[34] X.D. Fu, The superfamily of arginine/serine-rich splicing factors, RNA 1 (1995) 663-680.

[35] N. Proudfoot, Connecting transcription to messenger RNA processing, TIBS 25 (2000) 290-293. 